\title{
Resveratrol shortens the chronological lifespan of Saccharomyces cerevisiae by a pro- oxidant mechanism
}

\author{
Juan Carlos Canedo-Santos ${ }^{1}$, Iridian Mora-Martinez ${ }^{1}$, Ingrid Karina Gutierrez-Garcia ${ }^{1}$, Maria \\ Guadalupe Ramirez-Romero ${ }^{1}$ and Luis Alberto Madrigal-Perez ${ }^{1 *}$ \\ ${ }^{1}$ Tecnológico Nacional de México/ Instituto Tecnológico Superior de Ciudad Hidalgo, Av. \\ Ing. Carlos Rojas Gutiérrez \#2120, Ciudad Hidalgo, Michoacán, 61100, México. \\ " To whom correspondence should be addressed: Luis Alberto Madrigal-Perez: Tecnológico \\ Nacional de México/ Instituto Tecnológico Superior de Ciudad Hidalgo, Av. Ing. Carlos \\ Rojas Gutiérrez \#2120, Ciudad Hidalgo, Michoacán, 61100, México. \\ lmadrigal@ cdhidalgo.tecnm.mx. Phone: +52 (786) 154-9000. Fax: +52 (786) 154-9000.
}

\begin{abstract}
Resveratrol consumption has linked with normalization of the risk factors of some diseases such as colon cancer and type 2 diabetes. The antioxidant phenotype caused by resveratrol has recognized as a key piece in the health benefits exerted by this phytochemical. Although the antioxidant activity showed by resveratrol has attributed at the molecule per se, recent evidence indicates that the antioxidant effect occasioned by resveratrol could be associated with a pro-oxidant mechanism. The hypothesis that resveratrol inhibits complex III of the electron transport chain as its main target suggests that resveratrol increases reactive oxygen species (ROS) generation produces via reverse electron transport. This idea also explains that cells respond to the oxidative damage caused by resveratrol, inducing their antioxidant systems. The free radical theory of aging postulates that organisms age due to the accumulation of the harmful effects of ROS in cells. For these reasons, we hypothesize that resveratrol shortens the chronological life span (CLS) of Saccharomyces cerevisiae due to a pro-oxidant activity. Herein, we provide evidence that $100 \mu \mathrm{M}$ resveratrol supplementation at 5\% glucose: 1) shorted the CLS of CTT1 and YAP1 genes deleted strains; 2) decreased the $\mathrm{H}_{2} \mathrm{O}_{2}$ release in the WT strain, and maintain unaltered the $\mathrm{H}_{2} \mathrm{O}_{2}$ release in the $c t t 1 \Delta$ strain; 3) lessened exponential growth of $c t t 1 \Delta$ strain, which was reverted with the adding of GSH; 4) increased catalase activity in the WT strain, a phenotype that was not observed in the $c t t 1 \Delta$ strain. Altogether, these results indicate that resveratrol decreases CLS by a pro-oxidant mechanism.
\end{abstract}

\section{Keywords}

Oxidative stress; resveratrol; Saccharomyces cerevisiae; aging; antioxidant systems; catalase. 


\section{Introduction}

The resveratrol $\left(3,5,4^{\prime}\right.$-trihydroxy-trans-stilbene) is a polyphenol synthesized by some plants such as Vitis vinifera under biotic or abiotic stresses [1,2]. Resveratrol synthesis forms part of a chemical defense mechanism of plants to counteract or prevent infections $[3,4]$. Cytotoxic properties of resveratrol are well-documented and fit with its biological function in plants [5]. Another important effect exerted by resveratrol is the antioxidant, which has linked with the health benefits exerted by this compound [6]. However, the antioxidant properties of resveratrol are pro-survival to the cells and do not match with the toxic effect shown by resveratrol. Even the antioxidant molecular mechanism of resveratrol is not yet fully understood. The hypothesis that resveratrol inhibits the oxidative phosphorylation has gained attention in the last years and explains both its toxic and the antioxidant properties [7].

The inhibition of the electron transport chain (ETC) between complex I and complex III by resveratrol impair mitochondrial respiration [8]. The inhibition of the ETC by resveratrol could maintain the ubiquinone pool and complex I more reduced, increasing the chances of electron leaking and the formation of reactive oxygen species (ROS) [7]. In support of this idea, it has been reported that $5 \mathrm{mg} / \mathrm{kg}$ of resveratrol supplementation increases lipid peroxidation of the heart, liver, and kidney of rats [9]. Additionally, thyroid carcinoma THJ16T cell line supplemented with $100 \mu \mathrm{M}$ resveratrol increases superoxide anion production at the mitochondrial level [10]. The murine liver connective tissue cells GRX also augmented the ROS generation when they were supplemented with $50 \mu \mathrm{M}$ of resveratrol at $24 \mathrm{~h}$ and 120 $\mathrm{h}$ [11]. The increase of the ROS generation by resveratrol could induce the expression of the antioxidant systems as a defense mechanism to counteract this oxidative damage. In this regard, it has reported that Saccharomyces cerevisiae cells exposed to $5 \mu \mathrm{M}$ of resveratrol accumulated ROS and deletion of the antioxidant transcription factor gene YAP1 in these yeast cells increased cellular toxicity of resveratrol [12]. Also, in primary epidermal keratinocytes derived from human skin, $50 \mu \mathrm{M}$ of resveratrol supplementation triggered antioxidant systems transcription, dependent on the transcription factor Nrf2 [13]. Interestingly, resveratrol also augmented superoxide anion generation and intracellular ROS production in keratinocytes cells [13]. In accordance with the free radical theory of aging [14], resveratrol supplementation could shorten the chronological life span (CLS) of $S$. cerevisiae by a pro-oxidant mechanism.

Resveratrol also exerts phenotypes in a dose-dependent manner, fitting well with the hormetic behavior, as has been reported for cellular viability [15] and pro-oxidative properties [13]. Importantly, a diet-dependent effect of phenotypes exerted by resveratrol has also been documented [5]. For example, resveratrol exhibit a glucose-dependent effect in chronological aging [8], cellular viability [16], mitochondrial respiration [16], and hydrogen peroxide release [8] in S. cerevisiae. In the $\mathrm{Apc}^{\mathrm{Min}}$ mice, a model of colorectal carcinogenesis, resveratrol treatment $(0.00007 \%)$ decrease adenoma number per mouse in a high-fat diet but not in a standard diet [17]. However, the evidence linking the pro-oxidant properties of resveratrol with a glucose-dependent mechanism is still lacking.

For these reasons, this study aimed to demonstrate that resveratrol causes a pro-oxidant response that impact in chronological longevity, cell growth, and ROS generation in a glucose concentration-dependent manner. Herein, we provide evidence that resveratrol supplementation shortens CLS of $c t t 1 \triangle$ and yap $1 \triangle$ strains at $5 \%$ glucose. None effect was observed in CLS at $0.5 \%$ of glucose in $\mathrm{ctt} 1 \Delta$, yap $1 \Delta, \mathrm{hcm} 1 \Delta$, $\operatorname{sod} 2 \Delta$, and $m s n 2 \Delta$ deletant strains, with resveratrol supplementation. The deletion of the CTT1 gene reverts the decrease in the $\mathrm{H}_{2} \mathrm{O}_{2}$ release and the increase in catalase activity occasioned by resveratrol. At 5\% glucose, resveratrol supplementation diminishes the growth of the cttl $\Delta$ strain, and this phenotype was reestablished with the adding of reduced glutathione.

\section{Material and methods}

\section{Strains}


111

112

113

114

115

116

117

118

119

120

121

122

123

124

125

126

127

128

129

130

131

132

133

134

135

136

137

138

139

140

141

142

143

144

145

146

147

148

149

150

151

152

153

154

155

156

157

158

159

160

161

162

163
To perform the experiments was used S. cerevisiae BY4742 strain (MATa; his341; leu240;

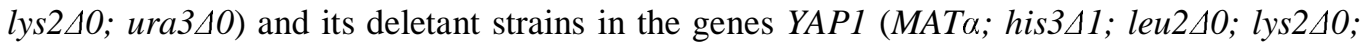

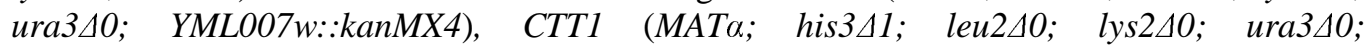

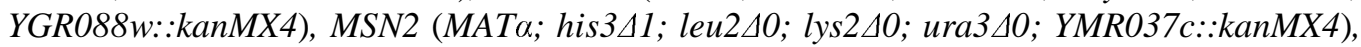

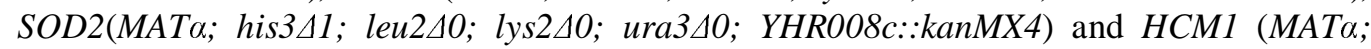

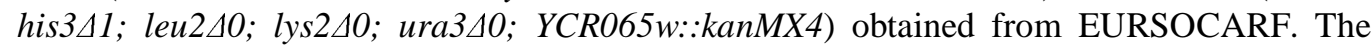
strains were maintained in yeast extract-peptone-dextrose (YPD) medium (1\% yeast extract, $2 \%$ casein peptone and $2 \%$ glucose), deletant strains were supplemented with geneticin (G418 disulfate salt solution, Sigma-Aldrich, St. Louis, MO, USA) at a final concentration of $200 \mu \mathrm{g} / \mathrm{mL}$.

\section{Chronological lifespan assay}

The CLS was determined according to Ramos-Gomez, Olivares-Marin, Canizal-Garcia, Gonzalez-Hernandez, Nava and Madrigal-Perez [8]. Briefly, the synthetic-complete (SC) medium was used to perform the CLS assay. It consisted of $0.18 \%$ yeast nitrogen base without amino acids, $0.5 \%$ ammonium sulfate, $0.2 \% \mathrm{KH}_{2} \mathrm{PO}_{4}, 1 \%$ drop-out mix without uracil, supplemented with $400 \mu \mathrm{g} / \mathrm{mL}$ of uracil. The mediums were supplemented with two different glucose concentrations $(0.5 \%$ and $5 \%)$ and five levels of resveratrol $(0,0.1,1,10$, 100 , and $1000 \mu \mathrm{M}$; trans-resveratrol $\geq 99 \%$ HPLC, Sigma-Aldrich) that were added at the beginning of the CLS assay. Afterward, $1 \mathrm{~mL}$ of the SC medium was inoculated with $1 \%$ of a fresh overnight culture of $S$. cerevisiae in a $15 \mathrm{~mL}$ conic tube. The $S$. cerevisiae cultures were grown at $30{ }^{\circ} \mathrm{C}$ with constant shaking at $250 \mathrm{rpm}$ for 15 days. Once three days of incubation were passed, aliquots of $5 \mu \mathrm{L}$ were taken and inoculated into $145 \mu \mathrm{L}$ YPD $2 \%$ glucose, every two days. Samples were placed in a 96-well plate and incubated at $30{ }^{\circ} \mathrm{C}$ for $24 \mathrm{~h}$ in a Varioskan Sky (Thermo-Scientific, Waltham, MA, USA) programmed with continuous shaking and readings at $600 \mathrm{~nm}$ each $60 \mathrm{~min}$. The survival percentage $(S n)$ was calculated according to equation 1 :

$S n=\frac{1}{2^{\left(\frac{\Delta t n}{t d}\right)}} \times 100$

Where $\Delta t n$ is the time shift (h), obtained by interpolation of D.O.600 $=0.5$ with a linear regression of the exponential phase of the growth curve, and $t d$ is the doubling time (h).

\section{Growth kinetics}

The cell growth was calculated using exponential growth as an indirect marker of cell division, as described by Olivares-Marin, Madrigal-Perez, Canizal-Garcia, GarciaAlmendarez, Gonzalez-Hernandez and Regalado-Gonzalez [18]. Growth kinetics were begun at O.D. $600 \bullet 0.1$ in a $25 \mathrm{~mL}$ shake flasks that contained $5 \mathrm{~mL}$ of YPD media supplemented with 5\% glucose and $100 \mu \mathrm{M}$ resveratrol. The shake flasks were incubated in a MaxQ 4000 incubated shaker (Thermo-Scientific) at $30{ }^{\circ} \mathrm{C}$ for 12 hours with shaking at $250 \mathrm{rpm}$. The growth was monitored, measuring the O.D. ${ }_{600}$ each hour. Data were analyzed using the statistical package GraphPad Prism 6.00 for Macintosh (GraphPad Software), fitting the growth kinetics with the exponential growth equation to obtain the specific growth rate $(\mu)$.

\section{Hydrogen peroxide release}

Hydrogen peroxide $\left(\mathrm{H}_{2} \mathrm{O}_{2}\right)$ release was quantified as an indicator of ROS production using the Amplex red hydrogen peroxide assay kit (Invitrogen, Waltham, MA, USA) following the manufacturer instructions. Briefly, exponential-growth phase S. cerevisiae cultures (O.D.600 0.6) grown in SC medium with $5 \%$ glucose were harvested at $5000 \mathrm{x} g$ for 5 minutes at $28^{\circ} \mathrm{C}$ and washed two times with $5 \mathrm{~mL}$ of sterile deionized water at $28{ }^{\circ} \mathrm{C}$. Then, cellular pellets were resuspended in $2 \mathrm{~mL}$ of assay buffer containing $20 \mathrm{mM}$ Tris- $\mathrm{HCl}, 0.5 \mathrm{mM}$ EDTA, $2 \%$ of ethanol at $\mathrm{pH} 7$. Finally, cells were placed into a 96-well plate at a density of $3 \times 10^{6}$ cells/well. The microplate was incubated at $30{ }^{\circ} \mathrm{C}$ with constant agitation for $30 \mathrm{~min}$. The 
164 basal release of $\mathrm{H}_{2} \mathrm{O}_{2}$ was measured at a wavelength of $560 \mathrm{~nm}$ with a microplate reader

165 (Varioskan Sky, Thermo-Scientific).

166

167

168

169

170

171

172

173

174

175

176

177

178

179

180

181

182

183

184

185

186

187

188

189

190

191

192

193

194

195

196

197

198

199

200

201

202

203

204

205

206

207

208

209

210

211

212

213

214

215

216

217

218

\section{Catalase activity}

Protein isolation for catalase activity assay was performed in exponential-growth phase $S$. cerevisiae cultures (O.D.600 0.6) grown in SC medium supplemented with $5 \%$ glucose and $100 \mu \mathrm{M}$ of resveratrol. Cultures were harvest at $13000 \mathrm{x} g$ for 3 minutes at $4{ }^{\circ} \mathrm{C}$ and resuspended in $500 \mu \mathrm{L}$ of sterile distilled water. Then, it was added $500 \mu \mathrm{L}$ of $0.7 \mathrm{~N}$ sodium hydroxide and incubated for 5 minutes at room temperature. Afterward, $0.2 \mathrm{~g}$ of glass beads $(0.2 \mathrm{~mm})$ were placed into the tubes and vortexing 1 minute and cooled into ice 2 minutes, repeating this two times. The solution was centrifuged at $5000 \mathrm{x} g$ for 1 minute, discarding the supernatant. The pellet was resuspended in a solution consisted of $100 \mathrm{mM}$ of potassium sodium, $5 \mathrm{mM}$ of EDTA, and $1 \mathrm{mM}$ of 2-mercaptoethanol. Next, the solution was centrifuged at $3500 \mathrm{x} g$ for $1 \mathrm{~min}$; the supernatant was used as the total protein isolation. For quantify catalase activity, $2 \mu \mathrm{L}$ of the total protein isolation was mixed with $0.5 \mu \mathrm{L}$ of $0.2 \mathrm{M} \mathrm{H}_{2} \mathrm{O}_{2}$ and placed into $\mu$ Drop plate. The plate was incubated at $25^{\circ} \mathrm{C}$ for 5 minutes in a Varioskan Sky microplate reader (Thermo-Scientific), recording the absorbance at $240 \mathrm{~nm}$ at the initial and final of the incubation period. The catalase activity was normalized with the total protein quantification.

\section{Statistical analyses}

The mean \pm standard deviation from at least three independent experiments was graphed. Means were compared using one-way ANOVA followed by a Dunnett multiple comparisons to analyze differences in the area under the curve from CLS assays and exponential growth. To analyze differences in the $\mathrm{H}_{2} \mathrm{O}_{2}$ release and catalase activity was used a two-tailed unpaired $t$-test. Statistical analyses were computed in the software GraphPad Prism 6.00 for Macintosh (GraphPad Software).

\section{Results}

Influence of resveratrol in the chronological aging of deletant strains in antioxidant systems To evaluate whether the shorten of CLS caused by resveratrol is coming from a pro-oxidant mechanism; we decided to measure the CLS in deletant strains in genes related to the antioxidant response of $S$. cerevisiae. The experiment was conducted with two glucose concentrations: $0.5 \%$ and $5 \%$, which promoting a respiratory and fermentative metabolism, respectively [18]. Additionally, it was used five levels of resveratrol in a logarithmic scale $(0.1,1,10,100$, and $1000 \mu \mathrm{M})$ to observe a dose-dependent effect.

The deletant strain of the gene encoding the cytosolic catalase $\mathrm{T}(C T T 1)$ showed a decrease in the CLS at 5\% glucose when it was supplemented with 100 and $1000 \mu \mathrm{M}$ of resveratrol in comparison with the $c t t 1 \Delta$ vehicle control (Fig. 1i-j). Low doses of resveratrol $(0.1,1$, and 10) have did not affect the CLS at 5\% glucose in the $c t t 1 \Delta$ strain (Fig. 1f-h). At $0.5 \%$ glucose, resveratrol did not modify the CLS when the CTT1 gene was deleted (Fig. 1a-e).

The Yap1p is a basic leucine zipper transcription factor, required for the response to the oxidative stress in S. cerevisiae [19]. The deletion of the YAPl gene occasioned a decrease in the CLS of S. cerevisiae grown at 5\% glucose, with 100 and $1000 \mu \mathrm{M}$ of resveratrol (Fig. 2ij). However, supplementation with $0.1,1$, and $10 \mu \mathrm{M}$ of resveratrol did not modify the CLS of the yap $1 \triangle$ strain at 5\% glucose (Fig. 2f-h). Under the low-glucose concentration (0.5\%), resveratrol supplementation did not affect the CLS of the yap $1 \triangle$ strain (Fig. 2a-e).

The transcriptional factors $\mathrm{Hcm} 1 \mathrm{p}$ and Msn2p participate in the response of $S$. cerevisiae to oxidative stress $[19,20]$. Nonetheless, the deletion of the genes $H C M 1$ and MSN2 did not change the CLS of S. cerevisiae at $0.5 \%$ or $5 \%$ glucose with any of the resveratrol concentrations tested (Fig. 3 and 4). Finally, the gene deletion of the mitochondrial manganese superoxide dismutase (SOD2) did not affect the CLS of S. cerevisiae at any 
glucose or resveratrol concentration (Fig. 5). Altogether, these results suggest that deletion

\section{$\mathrm{H}_{2} \mathrm{O}_{2}$ release in deletant strains in the antioxidant systems with resveratrol supplementation} To evaluate whether the decrease in CLS is related to a ROS production occasioned by resveratrol, a quantification of $\mathrm{H}_{2} \mathrm{O}_{2}$ release was carried out. For the following experiments, we decided to use only 100 of $\mu \mathrm{M}$ resveratrol, the lowest concentration that shortened the CLS and 5\% glucose, concentration in which is observed the CLS lessening. Resveratrol supplementation decreased the $\mathrm{H}_{2} \mathrm{O}_{2}$ release in comparison with the vehicle control in the WT strain (Fig. 6a); the same phenotype was displayed in the strains $h c m 1 \Delta, \operatorname{sod} 2 \Delta$, yap $1 \Delta$, and msn $2 \Delta$ (Fig. 6b-e). However, the $c t t 1 \Delta$ strain did not show a difference in comparison to vehicle control in the $\mathrm{H}_{2} \mathrm{O}_{2}$ release at $5 \%$ glucose (Fig. 6f). These data suggest the cytosolic catalase $\mathrm{T}$ is essential to decrease the $\mathrm{H}_{2} \mathrm{O}_{2}$ release prompted by resveratrol supplementation, and this could be impacting the CLS.

\section{Influence of glutathione and resveratrol supplementation in the growth of $\mathrm{ctt} 1 \Delta$ strain}

The reduced glutathione (GSH) is a well-known antioxidant molecule; we use it to assess whether a pro-oxidant mechanism exerts the detrimental effect of resveratrol upon $c t t 1 \triangle$ strain. Exponential growth was utilized as an indicator of resveratrol toxic influence [21]. Resveratrol supplementation decreased the growth of the $c t t 1 \Delta$ strain at 5\% of glucose (Fig. 7). As expected, the supplementation with $100 \mu \mathrm{M}$ of GSH reverted the negative phenotype exerted by resveratrol upon $c t t 1 \Delta$ growth (Fig. 7). This result indicates that the negative influence of resveratrol on $c t t 1 \Delta$ growth is due to a pro-oxidant effect, which is nullified by the GSH.

\section{Effect of resveratrol supplementation in the catalase activity of $\mathrm{S}$. cerevisiae}

The pro-oxidant properties of resveratrol might induce an antioxidant response of cells to counteract the oxidative stress provoked by this phytochemical. For this reason, the CTT1 gene deletion probably exacerbates the toxic effect of resveratrol. To prove this idea, we evaluate whether resveratrol supplementation increases the catalase activity of $S$. cerevisiae. Unsurprisingly, resveratrol supplementation augmented the catalase activity in comparison with the vehicle control in the WT strain (Fig. 8a). Importantly, the cttl $\triangle$ strain did not display the increase in the catalase activity induced by resveratrol supplementation (Fig. 8b). These data indicate that resveratrol promotes the activation of the catalase activity, possibly by a pro-oxidant mechanism.

\section{Discussion}

Resveratrol consumption has linked with normalization of the risk factors of some diseases such as colon cancer [17], type 2 diabetes [22], and non-alcoholic fatty liver [23]. Antioxidant activity has been associated with the health benefits showed by resveratrol [24]. However, it is not clear the mechanism by which resveratrol promotes antioxidant activity. The hypothesis that resveratrol inhibits the ETC as its main target, also suggests that resveratrol increases ROS generation produces via reverse electron transport [7]. This idea also explains that cells respond to the oxidative damage caused by resveratrol, inducing their antioxidant systems [5, 7]. The free radical theory of aging postulates that organisms age due to the accumulation of the harmful effects of ROS in cells [14]. For these reasons, we hypothesize that resveratrol shortens CLS of S. cerevisiae due to a pro-oxidant activity. Herein, we provide evidence that $100 \mu \mathrm{M}$ resveratrol supplementation at 5\% glucose: 1) shorted the CLS of CTT1 and YAP1 genes deleted strains; 2) decreased the $\mathrm{H}_{2} \mathrm{O}_{2}$ release in the WT strain, and maintain unaltered the $\mathrm{H}_{2} \mathrm{O}_{2}$ release in the $c t t 1 \Delta$ strain; 3) lessened exponential growth of $c t t 1 \Delta$ strain, which was reverted with the adding of GSH; 4) increased catalase activity in the WT strain, a phenotype that was not observed in the $c t t 1 \Delta$ strain. Altogether, these results indicate that resveratrol decreases CLS by a pro-oxidant mechanism. 
273 The role that resveratrol exerts on aging is not fully understanding yet. Early studies showed 274 that replicative life span (RLS) was extended with 10,100 , and $500 \mu \mathrm{M}$ of resveratrol in 275 PSY316AT S. cerevisiae cells grown in 2\% glucose YPD media [25]. Nonetheless, under the same conditions, it was reported that resveratrol $(10$ and $100 \mu \mathrm{M})$ did not change the RLS of S. cerevisiae BY4742 and W303 [26]. Interestingly, it was reported that resveratrol (100 $\mu$ M) shorted CLS in at $0.5 \%$ and $2 \%$ of glucose but not at $10 \%$ glucose in S. cerevisiae BY4742 strain grown in SC medium [8]. A decrease in CLS was also documented with the strain W303-1A grown in minimal medium supplemented with $2 \%$ glucose and $100 \mu \mathrm{M}$ resveratrol [27]. Importantly, CLS lessening was accompanied by an increase of ROS production promoted by resveratrol supplementation [27]. In this study, we found that $100 \mu \mathrm{M}$ of resveratrol shorted the CLS of the strains deleted in the genes CTT1 and YAP1 at 5\% glucose (Fig. 1 and 2). Noteworthy, that S. cerevisiae cells grown with $2 \%$ glucose or higher concentrations of glucose have a greater ROS production than cells grown at $0.5 \%$ glucose [28]. In this sense, the oxidative damage caused by resveratrol supplementation could be enhanced in 5\% glucose due to the ROS production provoked at this glucose concentration. In this sense, the deletion of the gene encoding the transcriptional factor Yap1p, required for oxidative stress tolerance and activated by $\mathrm{H}_{2} \mathrm{O}_{2}$, made more sensitive $S$. cerevisiae cells to resveratrol $(5 \mu \mathrm{M})$ toxicity in YPD and minimal media supplemented with $2 \%$ glucose [12]. Besides, supplementation with $5 \mu \mathrm{M}$ of resveratrol also augmented total ROS levels in $S$. cerevisiae [12]. Interestingly, Yap1p transcriptionally regulates the CTT1 gene [29], and both gene deletion shorted CLS of S. cerevisiae grown with $100 \mu \mathrm{M}$ resveratrol and 5\% glucose (Fig. 1 and 2). These results pointing out the importance of the cytosolic catalase $\mathrm{T}$ to counteract oxidative damage caused by resveratrol, suggesting a possible oxidativemechanism in the shorten of CLS by this phytochemical.

Several studies have reported the antioxidant response occasioned by resveratrol [30, 31]. Although the molecule has shown the capacity to reduce some oxidant molecules, it is not clear how resveratrol diminishes ROS levels within cells. Even, it has been observed that resveratrol antioxidant response depends on its concentration. For example, supplementation with $5 \mu \mathrm{M}$ of resveratrol augmented the total ROS levels in S. cerevisiae cultures, whereas cultures supplemented with $50 \mu \mathrm{M}$ of resveratrol decreased it [12]. Besides, also the glucose concentration has an impact on the antioxidant activity of resveratrol. For instance, the $\mathrm{H}_{2} \mathrm{O}_{2}$ release was diminished with $10 \mu \mathrm{M}$ of resveratrol at $10 \%$ glucose, while at $0.5 \%$ glucose, the same resveratrol concentration increased the $\mathrm{H}_{2} \mathrm{O}_{2}$ release [8]. We found that the deletion of the CTT1 gene reverted the diminution of the $\mathrm{H}_{2} \mathrm{O}_{2}$ release by resveratrol supplementation $(100 \mu \mathrm{M})\left(\right.$ Fig. 6). This result suggests that the $\mathrm{H}_{2} \mathrm{O}_{2}$ release lessening caused by resveratrol is related to a pro-oxidant mechanism countered by antioxidant systems like the cytosolic catalase T. The inhibition of the ETC by resveratrol could explain its pro-oxidant mechanism. Inhibitors of the ETC like resveratrol alter the electron flow rate changing the redox state of some site, lowering respiration rate, and raising ROS production in a given site of the ETC [32]. Resveratrol supplementation disrupts ETC activity between complex I [33] and complex III [21]. In this regard, resveratrol supplementation $(30,50$, and $100 \mu \mathrm{M})$ increased the basal mitochondrial respiration and decreased the $\mathrm{H}_{2} \mathrm{O}_{2}$ release at $10 \%$ of glucose in $S$. cerevisiae cultures [8]. On the contrary, at $0.5 \%$ of glucose $S$. cerevisiae cultures displayed a mitochondrial respiration inhibition and an increase in the $\mathrm{H}_{2} \mathrm{O}_{2}$ release with $10 \mu \mathrm{M}$ of resveratrol [8]. Overall, these data indicate that resveratrol promotes ROS generation via ETC inhibition.

We hypothesize that cells express their antioxidant systems to counteract the oxidative damage caused by resveratrol supplementation. Thus, the antioxidant activity of the cells is responsible for the antioxidant effect showing by resveratrol and not the compound per se. Supporting this idea, we found that resveratrol $(100 \mu \mathrm{M})$ increased catalase activity, and this phenotype was nullified in the $c t t 1 \Delta$ strain (Fig. 8). Besides, it was also reported that $100 \mu \mathrm{M}$ resveratrol stimulates catalase and superoxide dismutase activities in S. cerevisiae [27]. Additionally, expression of catalase was induced by resveratrol supplementation with 5, 25, 
50 and $100 \mu \mathrm{M}$ in normal human epidermal keratinocytes (NHEK) cells [13]. The prooxidant effect of resveratrol has also associated with its toxic influence upon cellular viability. For example, the addition of $25 \mathrm{mM}$ of the antioxidant molecule GSH, augmented the $\mathrm{IC}_{50}$ of resveratrol from $247 \mu \mathrm{M}$ to $747 \mu \mathrm{M}$ in NHEK cells, from $342.5 \mu \mathrm{M}$ to $1163 \mu \mathrm{M}$ in normal human dermal fibroblasts, and from $\geq 150 \mu \mathrm{M}$ to $445.3 \mu \mathrm{M}$ in HepG2 liver cells [13]. Likewise, we found that supplementation with $100 \mu \mathrm{M}$ of GSH rescued the $c t t 1 \Delta$ strain of the decrease in exponential growth caused by $100 \mu \mathrm{M}$ of resveratrol (Fig. 7). Altogether, these results indicate that resveratrol pro-oxidative properties play an important role in its toxic effect toward cell viability. Besides, these data suggest that cellular antioxidant systems are responsible for the antioxidant effect showed by resveratrol.

Overall, these results indicate that oxidative stress induced by resveratrol negatively impacts in exponential growth and CLS. Finally, it also suggests that antioxidant effects displayed by resveratrol are due to the cellular antioxidant response.

\section{Acknowledgments \\ Funding: Tecnológico Nacional de México supported LAMP (grant number 5388.19-P).}

\section{Conflict of interest statement}

The authors declare that they have no conflict of interest.

\section{References}

[1] P. Jeandet, R. Bessis, M. Sbaghi, P. Meunier, Production of the phytoalexin resveratrol by grapes as a response to Botrytis attack under natural conditions, Journal of Phytopathology, 143 (1995) 135-139.

[2] P. Langcake, R.J. Pryce, The production of resveratrol by Vitis Vinifera and other members of the Vitaceae as a response to infection or injury, Physiological Plant Pathology, 9 (1976) 77-86.

[3] M. Adrian, P. Jeandet, J. Veneau, L.A. Weston, R. Bessis, Biological activity of resveratrol, a stilbenic compound from grapevines, against Botrytis cinerea, the causal agent for gray mold, J Chem Ecol, 23 (1997) 1689-1702.

[4] P. Jeandet, C. Hebrard, M.A. Deville, S. Cordelier, S. Dorey, A. Aziz, J. Crouzet, Deciphering the role of phytoalexins in plant-microorganism interactions and human health, Molecules, 19 (2014) 18033-18056.

[5] I.K. Olivares-Marin, J.C. Gonzalez-Hernandez, L.A. Madrigal-Perez, Resveratrol cytotoxicity is energy-dependent, Journal of food biochemistry, 43 (2019) e13008.

[6] Z. Ungvari, W.E. Sonntag, R. de Cabo, J.A. Baur, A. Csiszar, Mitochondrial protection by resveratrol, Exercise and sport sciences reviews, 39 (2011) 128-132.

[7] L.A. Madrigal-Perez, M. Ramos-Gomez, Resveratrol inhibition of cellular respiration: new paradigm for an old mechanism, International journal of molecular sciences, 17 (2016) 368.

[8] M. Ramos-Gomez, I.K. Olivares-Marin, M. Canizal-Garcia, J.C. Gonzalez-Hernandez, G.M. Nava, L.A. Madrigal-Perez, Resveratrol induces mitochondrial dysfunction and decreases chronological life span of Saccharomyces cerevisiae in a glucose-dependent manner, Journal of bioenergetics and biomembranes, 49 (2017) 241-251.

[9] W. Gadacha, M. Ben-Attia, D. Bonnefont-Rousselot, E. Aouani, N. Ghanem-Boughanmi, Y. Touitou, Resveratrol opposite effects on rat tissue lipoperoxidation: pro-oxidant during day-time and antioxidant at night, Redox report : communications in free radical research, 14 (2009) 154-158.

[10] X. Zheng, B. Jia, X.T. Tian, X. Song, M.L. Wu, Q.Y. Kong, H. Li, J. Liu, Correlation of Reactive Oxygen Species Levels with Resveratrol Sensitivities of Anaplastic Thyroid Cancer Cells, Oxidative medicine and cellular longevity, 2018 (2018) 6235417.

[11] L.A. Martins, B.P. Coelho, G. Behr, L.F. Pettenuzzo, I.C. Souza, J.C. Moreira, R. Borojevic, C. Gottfried, F.C. Guma, Resveratrol induces pro-oxidant effects and time- 
382 dependent resistance to cytotoxicity in activated hepatic stellate cells, Cell biochemistry and 383 biophysics, 68 (2014) 247-257.

[12] X. Escote, M. Miranda, S. Menoyo, B. Rodriguez-Porrata, D. Carmona-Gutierrez, H. Jungwirth, F. Madeo, R.R. Cordero, A. Mas, F. Tinahones, J. Clotet, J. Vendrell, Resveratrol induces antioxidant defence via transcription factor Yap1p, Yeast, 29 (2012) 251-263.

[13] A. Plauth, A. Geikowski, S. Cichon, S.J. Wowro, L. Liedgens, M. Rousseau, C. Weidner, L. Fuhr, M. Kliem, G. Jenkins, S. Lotito, L.J. Wainwright, S. Sauer, Hormetic shifting of redox environment by pro-oxidative resveratrol protects cells against stress, Free Radical Biology and Medicine, 99 (2016) 608-622.

[14] D. Harman, Aging: a theory based on free radical and radiation chemistry., J Gerontol., 11 (1956) 298-300.

[15] E.J. Calabrese, M.P. Mattson, V. Calabrese, Resveratrol commonly displays hormesis: occurrence and biomedical significance, Human \& experimental toxicology, 29 (2010) 9801015.

[16] L.A. Madrigal-Perez, M. Canizal-Garcia, J.C. Gonzalez-Hernandez, R. ReynosoCamacho, G.M. Nava, M. Ramos-Gomez, Energy-dependent effects of resveratrol in Saccharomyces cerevisiae, Yeast, 33 (2016) 227-234.

[17] H. Cai, E. Scott, A. Kholghi, C. Andreadi, A. Rufini, A. Karmokar, R.G. Britton, E. Horner-Glister, P. Greaves, D. Jawad, M. James, L. Howells, T. Ognibene, M. Malfatti, C. Goldring, N. Kitteringham, J. Walsh, M. Viskaduraki, K. West, A. Miller, D. Hemingway, W.P. Steward, A.J. Gescher, K. Brown, Cancer chemoprevention: Evidence of a nonlinear dose response for the protective effects of resveratrol in humans and mice, Science translational medicine, 7 (2015) 298ra117.

[18] I.K. Olivares-Marin, L.A. Madrigal-Perez, M. Canizal-Garcia, B.E. Garcia-Almendarez, J.C. Gonzalez-Hernandez, C. Regalado-Gonzalez, Interactions between carbon and nitrogen sources depend on RIM15 and determine fermentative or respiratory growth in Saccharomyces cerevisiae, Appl Microbiol Biotechnol, 102 (2018) 4535-4548.

[19] C. Rodrigues-Pousada, F. Devaux, S.M. Caetano, C. Pimentel, S. da Silva, A.C. Cordeiro, C. Amaral, Yeast AP-1 like transcription factors (Yap) and stress response: a current overview, Microbial cell, 6 (2019) 267-285.

[20] M.J. Rodriguez-Colman, G. Reverter-Branchat, M.A. Sorolla, J. Tamarit, J. Ros, E. Cabiscol, The forkhead transcription factor $\mathrm{Hcm} 1$ promotes mitochondrial biogenesis and stress resistance in yeast, The Journal of biological chemistry, 285 (2010) 37092-37101.

[21] L.A. Madrigal-Perez, G.M. Nava, J.C. Gonzalez-Hernandez, M. Ramos-Gomez, Resveratrol increases glycolytic flux in Saccharomyces cerevisiae via a SNF1-dependet mechanism, Journal of bioenergetics and biomembranes, 47 (2015) 331-336.

[22] A. Hoseini, G. Namazi, A. Farrokhian, Z. Reiner, E. Aghadavod, F. Bahmani, Z. Asemi, The effects of resveratrol on metabolic status in patients with type 2 diabetes mellitus and coronary heart disease, Food \& function, 10 (2019) 6042-6051.

[23] M.M. Tiao, Y.J. Lin, H.R. Yu, J.M. Sheen, I.C. Lin, Y.J. Lai, Y.L. Tain, L.T. Huang, C.C. Tsai, Resveratrol ameliorates maternal and post-weaning high-fat diet-induced nonalcoholic fatty liver disease via renin-angiotensin system, Lipids in health and disease, 17 (2018) 178.

[24] F.R. Jardim, F.T. de Rossi, M.X. Nascimento, R.G. da Silva Barros, P.A. Borges, I.C. Prescilio, M.R. de Oliveira, Resveratrol and Brain Mitochondria: a Review, Molecular neurobiology, 55 (2018) 2085-2101.

[25] K.T. Howitz, K.J. Bitterman, H.Y. Cohen, D.W. Lamming, S. Lavu, J.G. Wood, R.E. Zipkin, P. Chung, A. Kisielewski, L.L. Zhang, B. Scherer, D.A. Sinclair, Small molecule activators of sirtuins extend Saccharomyces cerevisiae lifespan, Nature, 425 (2003) 191-196. [26] M. Kaeberlein, T. McDonagh, B. Heltweg, J. Hixon, E.A. Westman, S.D. Caldwell, A. Napper, R. Curtis, P.S. DiStefano, S. Fields, A. Bedalov, B.K. Kennedy, Substrate-specific activation of sirtuins by resveratrol, Journal of Biological Chemistry, 280 (2005) 1703817045. 
435 [27] I. Orlandi, G. Stamerra, M. Strippoli, M. Vai, During yeast chronological aging 436 resveratrol supplementation results in a short-lived phenotype Sir2-dependent, Redox 437 biology, 12 (2017) 745-754.

438 [28] M.F. Tello-Padilla, A.Y. Perez-Gonzalez, M. Canizal-Garcia, J.C. Gonzalez-Hernandez, 439 C. Cortes-Rojo, I.K. Olivares-Marin, L.A. Madrigal-Perez, Glutathione levels influence 440 chronological life span of Saccharomyces cerevisiae in a glucose-dependent manner, Yeast, 44135 (2018) 387-396.

442 [29] N. Nakazawa, H. Yanata, N. Ito, E. Kaneta, K. Takahashi, Oxidative stress tolerance of a 443 spore clone isolated from Shirakami kodama yeast depends on altered regulation of Msn2 444 leading to enhanced expression of ROS-degrading enzymes, The Journal of general and 445 applied microbiology, 64 (2018) 149-157.

446 [30] P. Silva, A. Sureda, J.A. Tur, P. Andreoletti, M. Cherkaoui-Malki, N. Latruffe, How 447 efficient is resveratrol as an antioxidant of the Mediterranean diet, towards alterations during 448 the aging process?, Free radical research, 53 (2019) 1101-1112.

449 [31] B.A.Q. Gomes, J.P.B. Silva, C.F.R. Romeiro, S.M. Dos Santos, C.A. Rodrigues, P.R. 450 Goncalves, J.T. Sakai, P.F.S. Mendes, E.L.P. Varela, M.C. Monteiro, Neuroprotective 451 Mechanisms of Resveratrol in Alzheimer's Disease: Role of SIRT1, Oxidative medicine and 452 cellular longevity, 2018 (2018) 8152373.

453 [32] M.D. Brand, Mitochondrial generation of superoxide and hydrogen peroxide as the 454 source of mitochondrial redox signaling, Free Radical Biology and Medicine, 100 (2016) 1445531.

456 [33] A.C. Moreira, A.M. Silva, M.S. Santos, V.A. Sardao, Resveratrol affects differently rat 457 liver and brain mitochondrial bioenergetics and oxidative stress in vitro: investigation of the 458 459 role of gender, Food and Chemical Toxicology, 53 (2013) 18-26.

\section{Figure legends}

Fig 1. Effect of deletion of CTT1 gene on the CLS of S. cerevisiae supplemented with resveratrol. The CLS was assayed in SC-medium using two glucose concentrations $(0.5 \%$ and $5 \%)$ and five levels of resveratrol $(0,0.1,1,10,100$, and $1000 \mu \mathrm{M})$. a)-e) CLS of $c t t 1 \Delta$ strain grown with $0.5 \%$ glucose and with $0,0.1,1,10,100$, and $1000 \mu \mathrm{M}$ of resveratrol, respectively; g)-j) CLS of $c t t 1 \Delta$ strain grown with 5\% glucose and with $0,0.1,1,10,100$, and $1000 \mu \mathrm{M}$ of resveratrol, respectively; k)-l) CLS comparison between $c t t 1 \triangle$ and WT strain at $0.5 \%$, and $5 \%$ glucose, respectively; m)-n) Represents the area under the curve (AUC) of CLS assays at $0.5 \%$ and $5 \%$, respectively. The AUC survival was calculated from the data of the percentage of cellular viability vs. time using the trapezoidal rule in the GraphPad Prism 6.00. The results represent mean values \pm standard deviation from four to five independent experiments, which include mean values of three technical repetitions. Statistical analyses were performed using one-way ANOVA followed by Dunnett's test vs. ctt1 $\triangle$ (vehicle control), ** $P \leq 0.01 ; * * * P \leq 0.001$.

Fig 2. Influence of YAPl gene deletion on the CLS of S. cerevisiae supplemented with resveratrol. The CLS was assayed in SC-medium using two glucose concentrations $(0.5 \%$ and $5 \%)$ and five levels of resveratrol $(0,0.1,1,10,100$, and $1000 \mu \mathrm{M})$. a)-e) CLS of yap1 $\Delta$ strain grown with $0.5 \%$ glucose and with $0,0.1,1,10,100$, and $1000 \mu \mathrm{M}$ of resveratrol, respectively; g)-j) CLS of yap1 $\triangle$ strain grown with 5\% glucose and with $0,0.1,1,10,100$, and $1000 \mu \mathrm{M}$ of resveratrol, respectively; k)-l) CLS comparison between yap1 $\triangle$ and WT strain at $0.5 \%$, and $5 \%$ glucose, respectively; m)-n) Represents the area under the curve (AUC) of CLS assays at $0.5 \%$ and $5 \%$, respectively. The AUC survival was calculated from the data of the percentage of cellular viability vs. time using the trapezoidal rule in the GraphPad Prism 6.00. The results represent mean values \pm standard deviation from four to five independent experiments, which include mean values of three technical repetitions. Statistical analyses were performed using one-way ANOVA followed by Dunnett's test $v s$. yap $1 \triangle$ (vehicle control), ${ }^{*} P \leq 0.05 ; * * P \leq 0.01$. 
Fig 3. Impact of HCM1 gene deletion on the CLS of $S$. cerevisiae supplemented with resveratrol. The CLS was assayed in SC-medium using two glucose concentrations $(0.5 \%$ and $5 \%)$ and five levels of resveratrol $(0,0.1,1,10,100$, and $1000 \mu \mathrm{M})$. a)-e) CLS of $h \mathrm{~cm} 1 \Delta$ strain grown with $0.5 \%$ glucose and with $0,0.1,1,10,100$, and $1000 \mu \mathrm{M}$ of resveratrol, respectively; g)-j) CLS of $h c m l \Delta$ strain grown with 5\% glucose and with $0,0.1,1,10,100$, and $1000 \mu \mathrm{M}$ of resveratrol, respectively; k)-l) CLS comparison between $h \mathrm{cml} 1 \Delta$ and WT strain at $0.5 \%$, and $5 \%$ glucose, respectively; m)-n) Represents the area under the curve (AUC) of CLS assays at $0.5 \%$ and $5 \%$, respectively. The AUC survival was calculated from the data of the percentage of cellular viability vs. time using the trapezoidal rule in the GraphPad Prism 6.00. The results represent mean values \pm standard deviation from four to five independent experiments, which include mean values of three technical repetitions. Statistical analyses were performed using one-way ANOVA followed by Dunnett's test $v s$. hcml $\triangle$ (vehicle control).

Fig 4. Effect of MSN2 gene deletion on the CLS of S. cerevisiae supplemented with resveratrol. The CLS was assayed in SC-medium using two glucose concentrations $(0.5 \%$ and $5 \%)$ and five levels of resveratrol $(0,0.1,1,10,100$, and $1000 \mu \mathrm{M})$. a)-e) CLS of $m s n 2 \Delta$ strain grown with $0.5 \%$ glucose and with $0,0.1,1,10,100$, and $1000 \mu \mathrm{M}$ of resveratrol, respectively; g)-j) CLS of $m s n 2 \Delta$ strain grown with 5\% glucose and with $0,0.1,1,10,100$, and $1000 \mu \mathrm{M}$ of resveratrol, respectively; k)-l) CLS comparison between $m s n 2 \Delta$ and WT strain at $0.5 \%$, and $5 \%$ glucose, respectively; m)-n) Represents the area under the curve (AUC) of CLS assays at $0.5 \%$ and $5 \%$, respectively. The AUC survival was calculated from the data of the percentage of cellular viability vs. time using the trapezoidal rule in the GraphPad Prism 6.00. The results represent mean values \pm standard deviation from four to five independent experiments, which include mean values of three technical repetitions. Statistical analyses were performed using one-way ANOVA followed by Dunnett's test vs. $m s n 2 \Delta$ (vehicle control).

Fig 5. Influence of deletion SOD2 gene on the CLS of $S$. cerevisiae supplemented with resveratrol. The CLS was assayed in SC-medium using two glucose concentrations $(0.5 \%$ and $5 \%)$ and five levels of resveratrol $(0,0.1,1,10,100$, and $1000 \mu \mathrm{M})$. a)-e) CLS of $\operatorname{sod} 2 \Delta$ strain grown with $0.5 \%$ glucose and with $0,0.1,1,10,100$, and $1000 \mu \mathrm{M}$ of resveratrol, respectively; g)-j) CLS of $\operatorname{sod} 2 \triangle$ strain grown with $5 \%$ glucose and with $0,0.1,1,10,100$, and $1000 \mu \mathrm{M}$ of resveratrol, respectively; k)-l) CLS comparison between $\operatorname{sod} 2 \Delta$ and WT strain at $0.5 \%$, and $5 \%$ glucose, respectively; m)-n) Represents the area under the curve (AUC) of CLS assays at $0.5 \%$ and $5 \%$, respectively. The AUC survival was calculated from the data of the percentage of cellular viability vs. time using the trapezoidal rule in the GraphPad Prism 6.00. The results represent mean values \pm standard deviation from four to five independent experiments, which include mean values of three technical repetitions. Statistical analyses were performed using one-way ANOVA followed by Dunnett's test vs. $\operatorname{sod} 2 \Delta$ (vehicle control).

Fig 6. Effect of the deletion of genes CTT1, YAP1, HCM1, MSN2, and SOD2 upon $\mathrm{H}_{2} \mathrm{O}_{2}$ release in $S$. cerevisiae supplemented with resveratrol. For the $\mathrm{H}_{2} \mathrm{O}_{2}$ release quantification, were utilized exponential-growth phase $S$. cerevisiae cultures (O.D.600 $\sim 0.6$ ) grown in SC medium supplemented with $5 \%$ glucose. The amplex red hydrogen peroxide assay kit was used for the determination of the $\mathrm{H}_{2} \mathrm{O}_{2}$ release. a)-f) Comparison of $\mathrm{H}_{2} \mathrm{O}_{2}$ release between vehicle control and cells supplemented with $100 \mu \mathrm{M}$ of resveratrol in the WT, ctt1 $1 \Delta$, yap $1 \Delta$, $h c m 1 \Delta, m s n 2 \Delta$, and $\operatorname{sod} 2 \Delta$ strains, respectively. The results represent mean values \pm standard deviation from three independent experiments. Means were compared with a twotailed unpaired $t$-test $(* P<0.05 ; * * P<0.01)$. 
542 Fig 7. Impact of resveratrol in the exponential growth of $c t t 1 \triangle$ strain supplemented with 543 reduced glutathione. The specific growth rate was used as an indicator of the exponential 544 growth of $c t t 1 \Delta$ grown in YPD medium at $5 \%$ glucose. The results represent mean values \pm 545 standard deviation from four to five independent experiments, which include mean values of 546 three technical repetitions. Statistical analyses were performed using one-way ANOVA 547 followed by Dunnett's test vs. cttl $\triangle$ (vehicle control).

Fig 8. Effect of resveratrol supplementation and $C T T 1$ gene deletion on catalase activity of $S$. cerevisiae. Catalase activity was measured in exponential-growth phase $S$. cerevisiae cultures (O.D.600 0.6) grown in SC medium supplemented with 5\% glucose. a)-b) Catalase activity comparison between vehicle control and cells supplemented with $100 \mu \mathrm{M}$ of resveratrol in the WT, and $c t t l$ strains, respectively. The results represent mean values \pm standard deviation from three to four independent experiments. Means were compared with a two-tailed unpaired $t$-test $(* * P<0.01)$. 


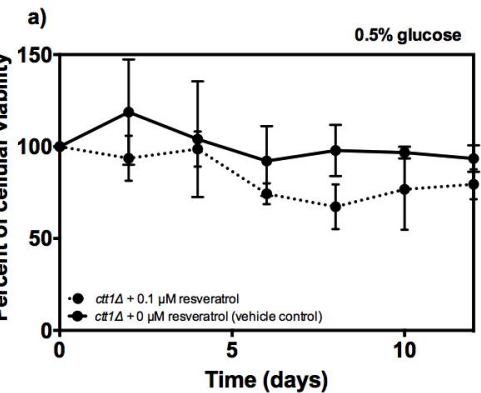

b)
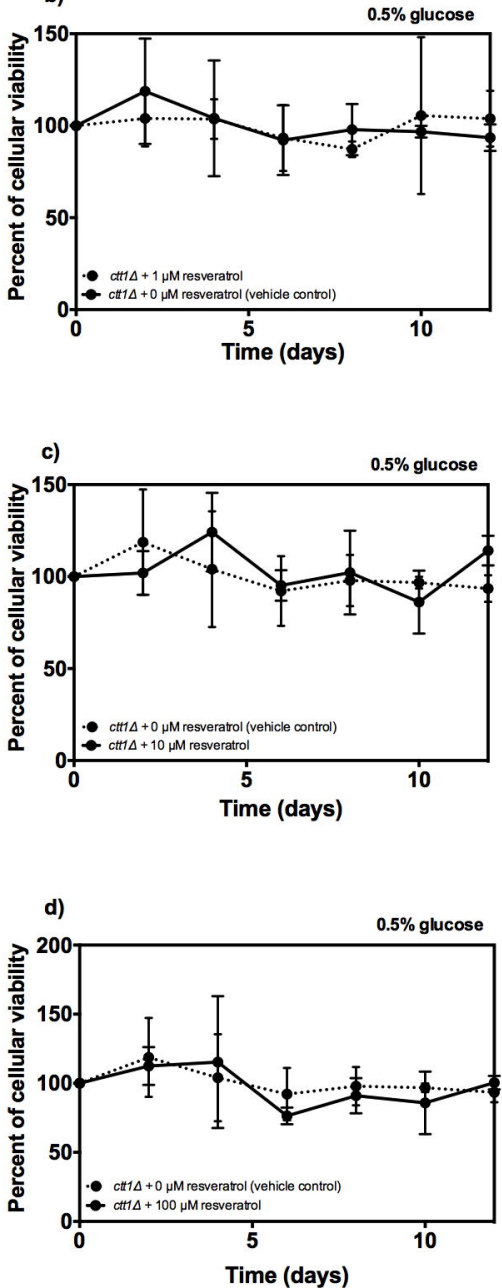
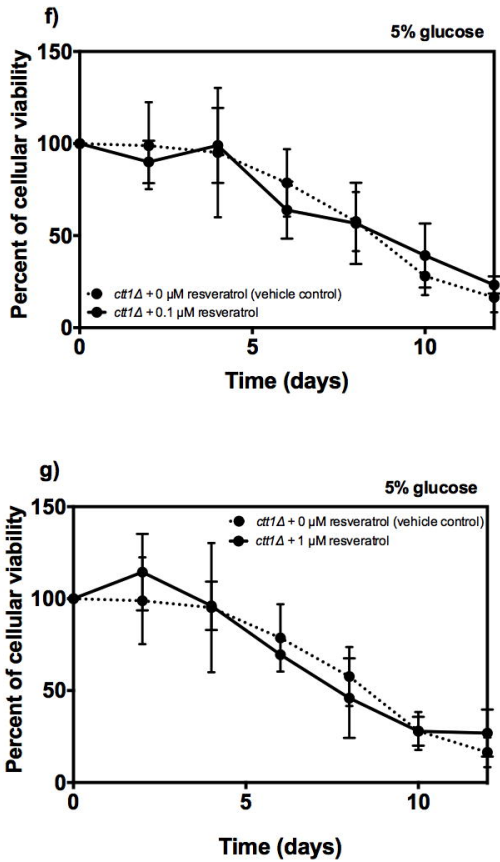

h)
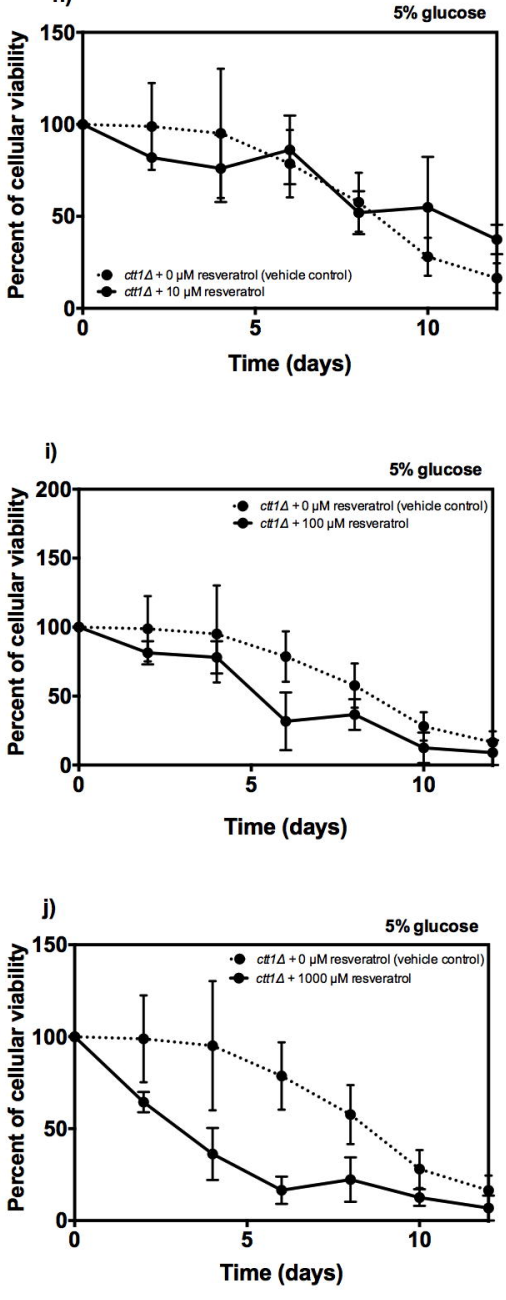
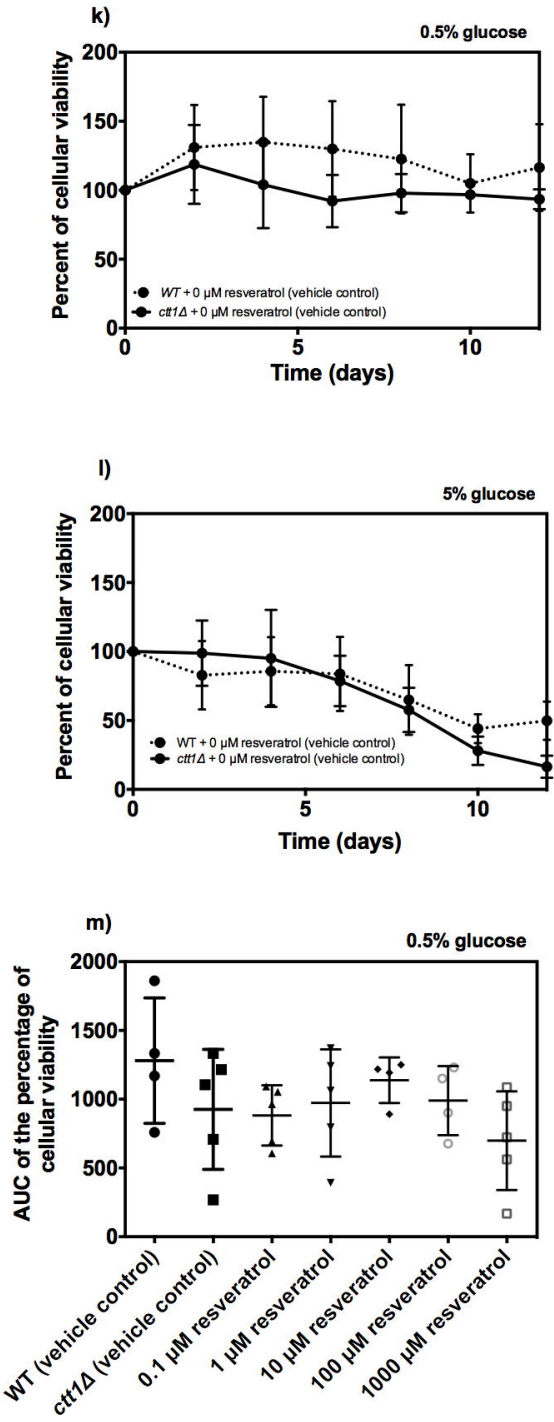

n)
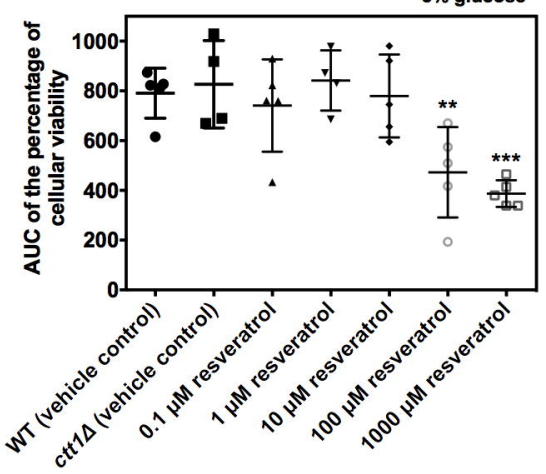
a)

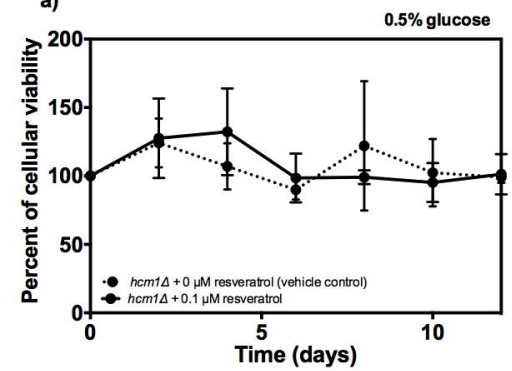

b)

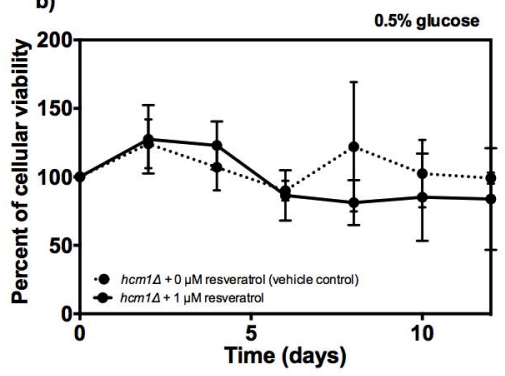

c)

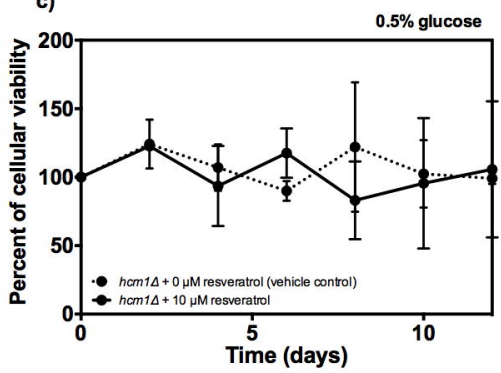

d)

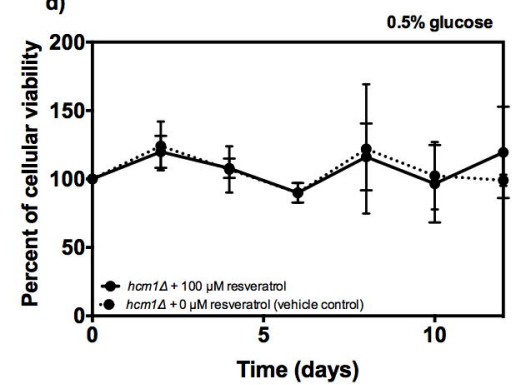

e)
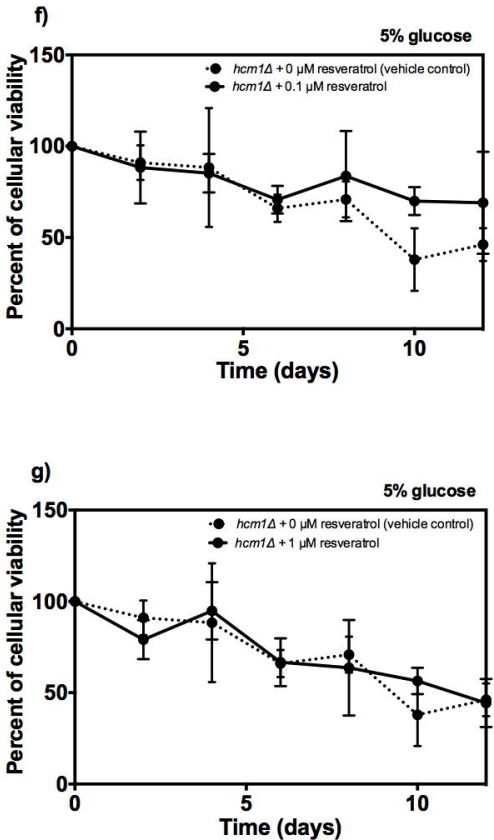

h)

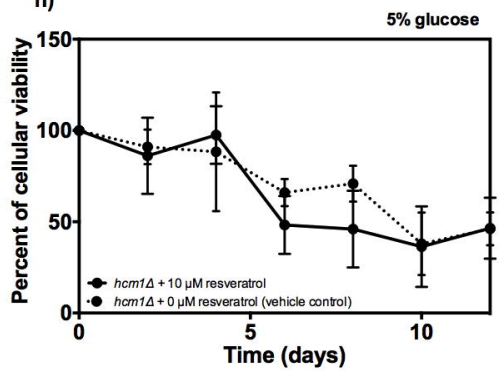

i)

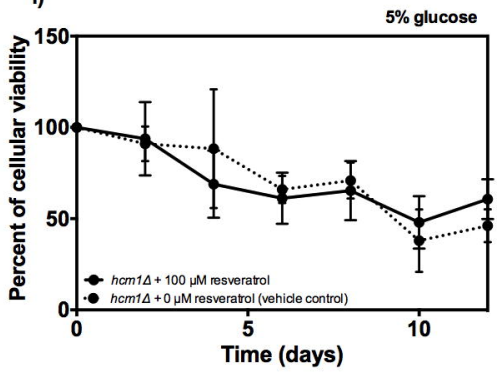

j)

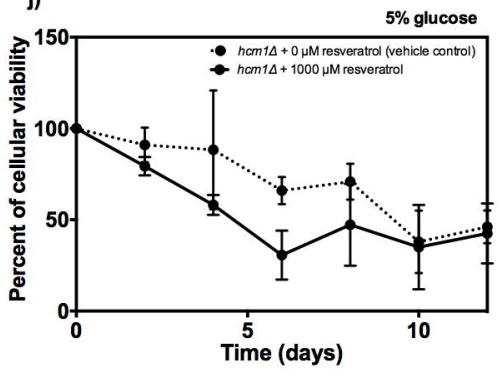

k)
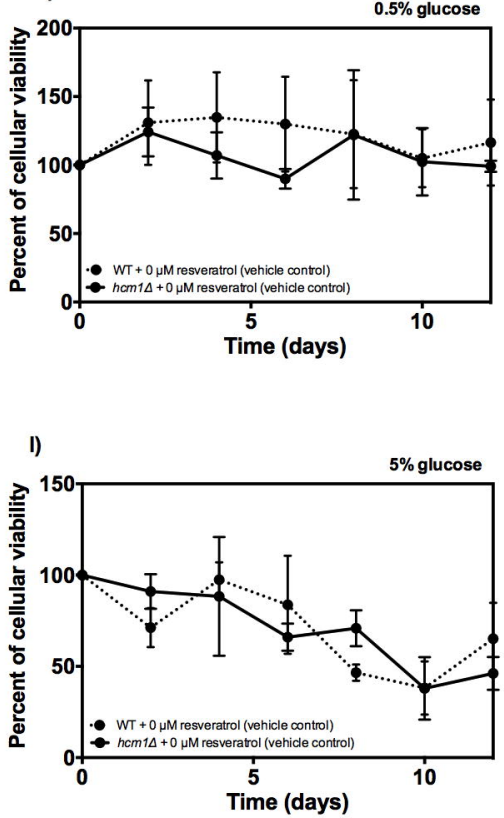

m)

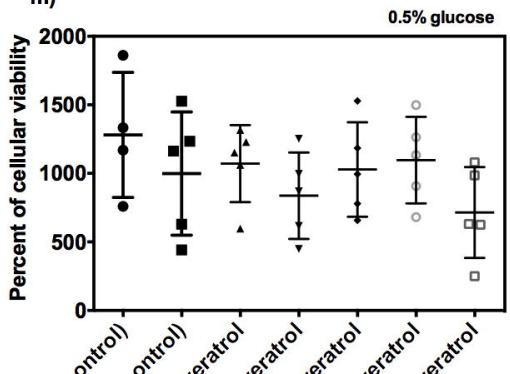

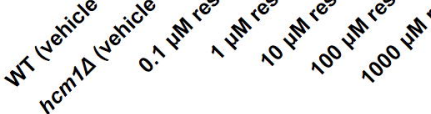

n)

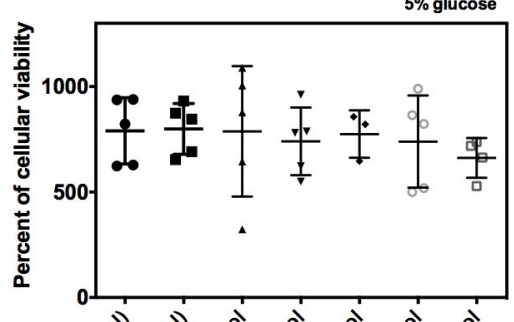

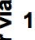
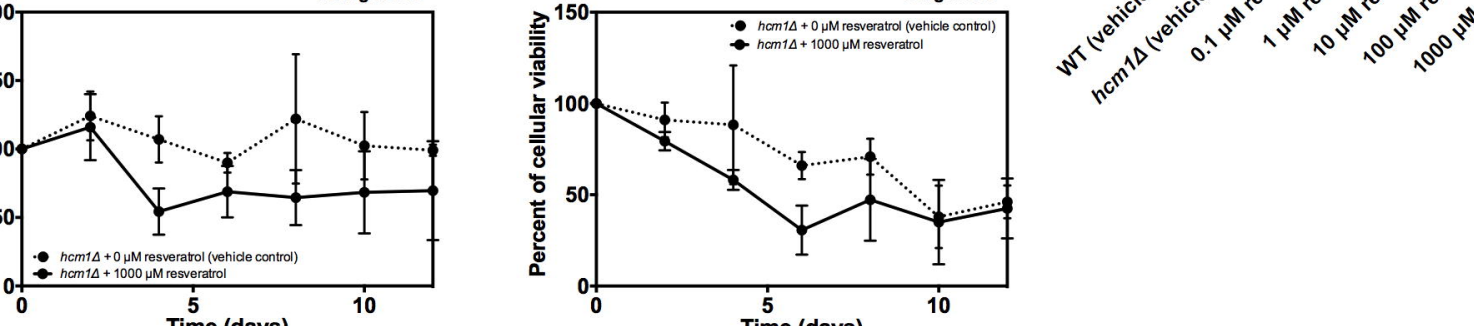
a)

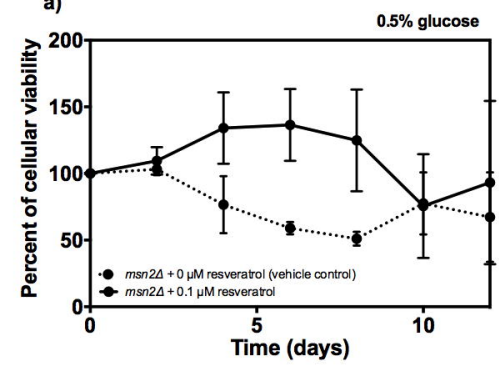

b)

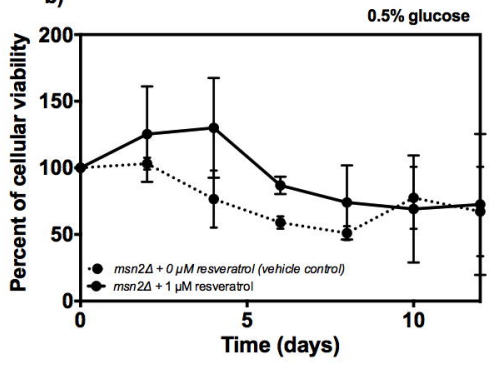

c)

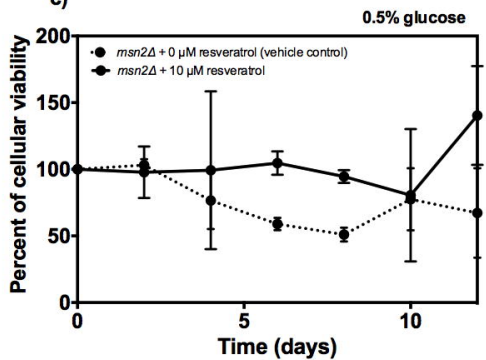

d)

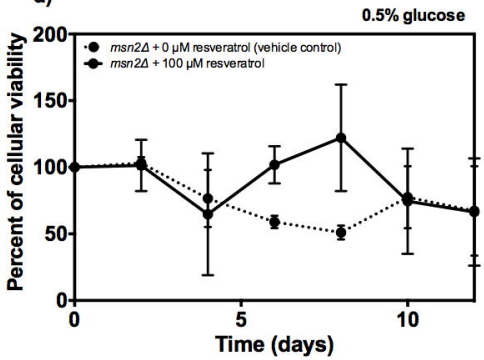

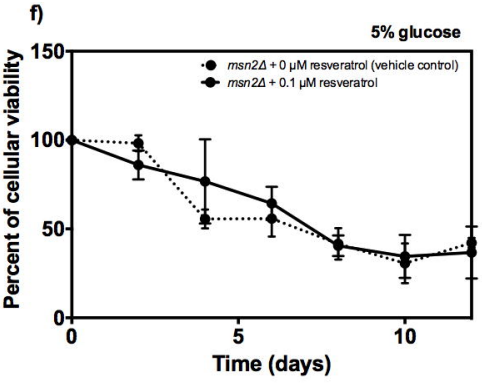

g)

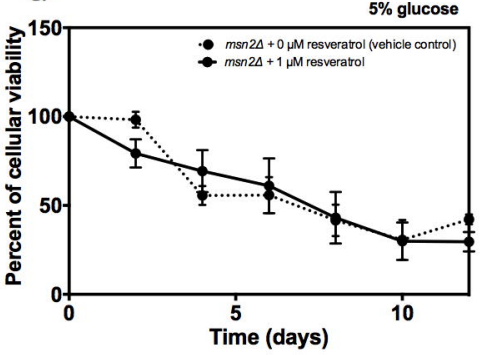

h)
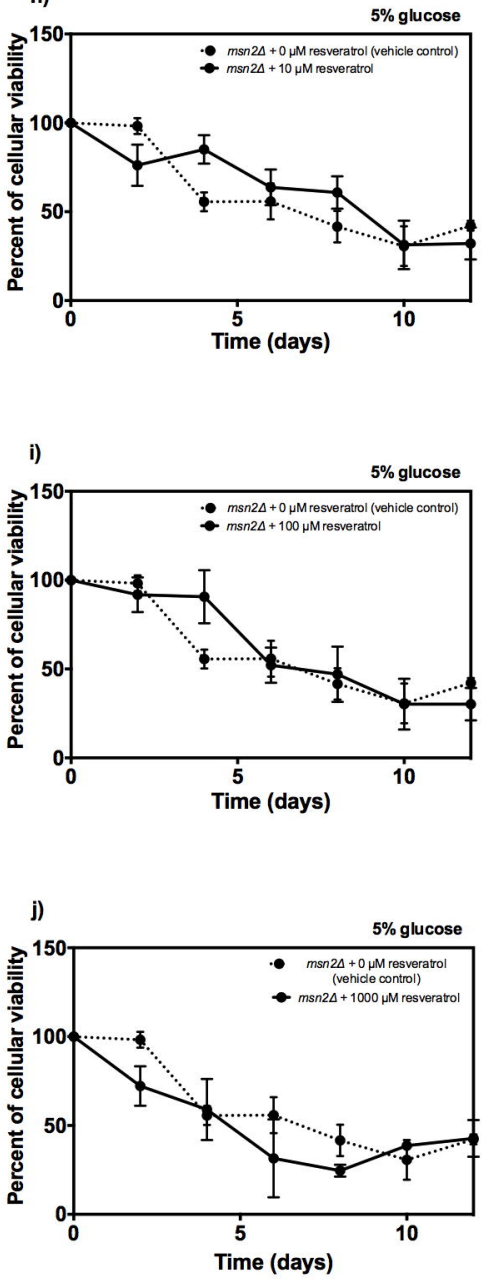
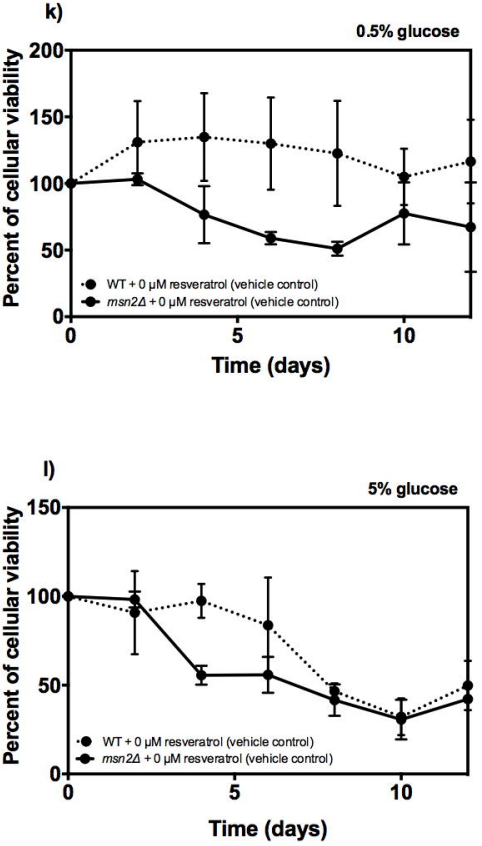

m)

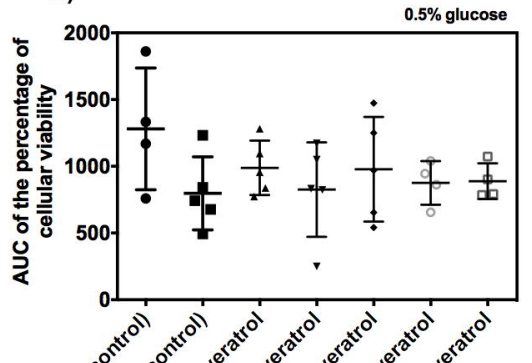

n)
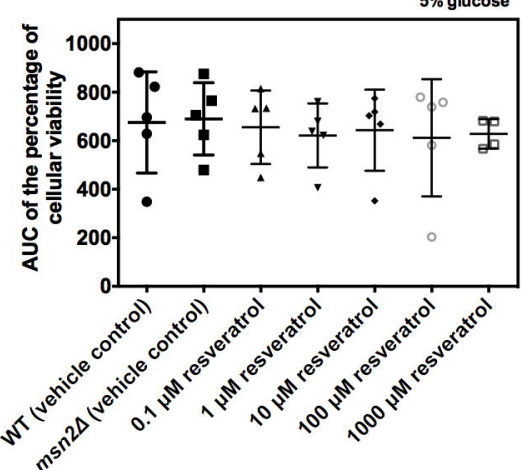


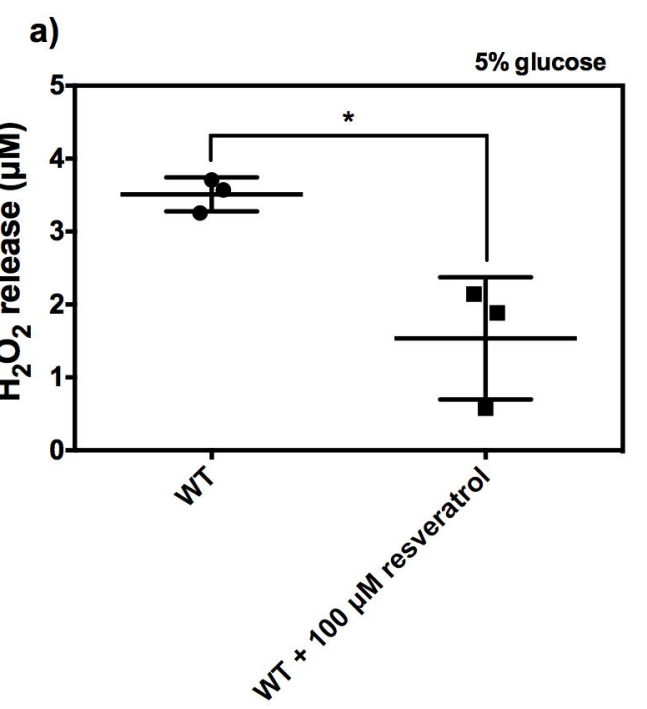

b)

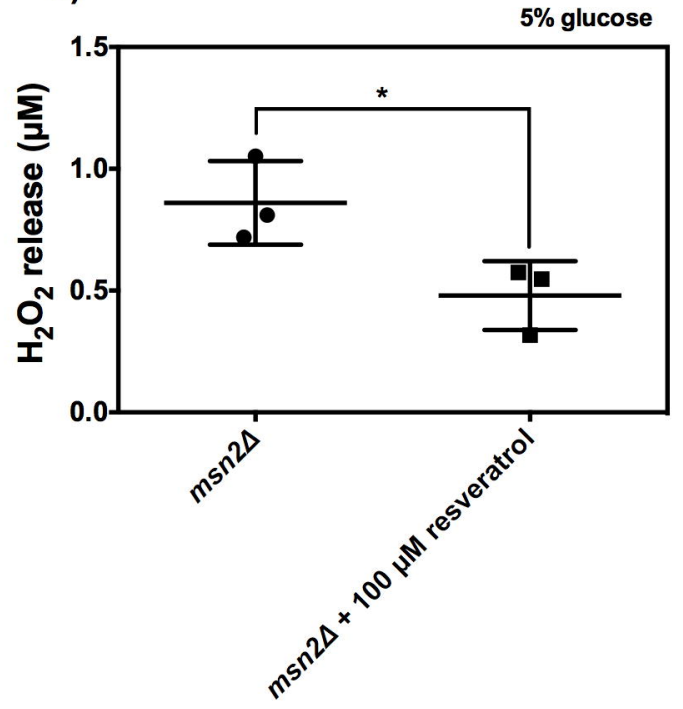

c)

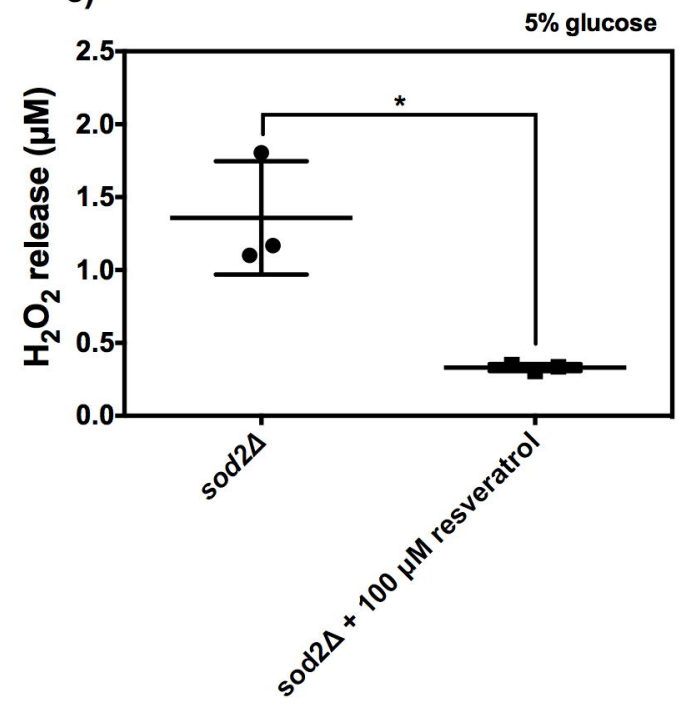

e)

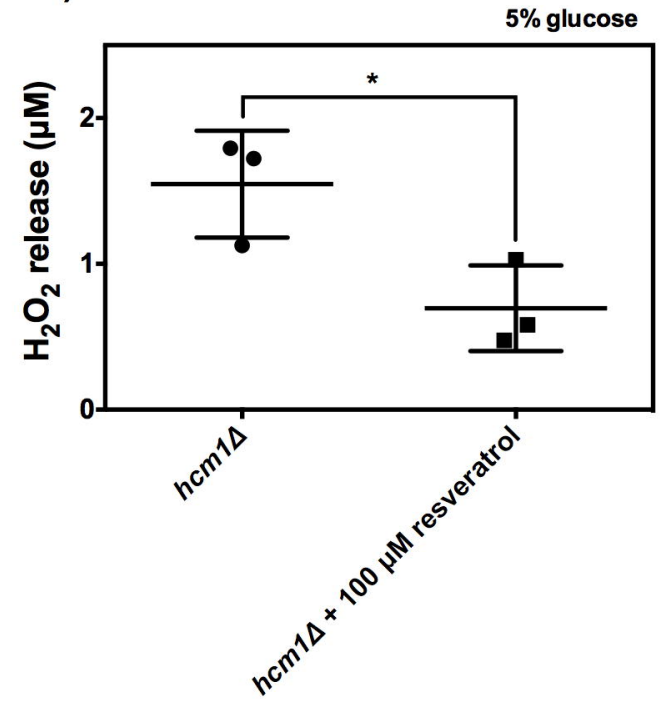

d)

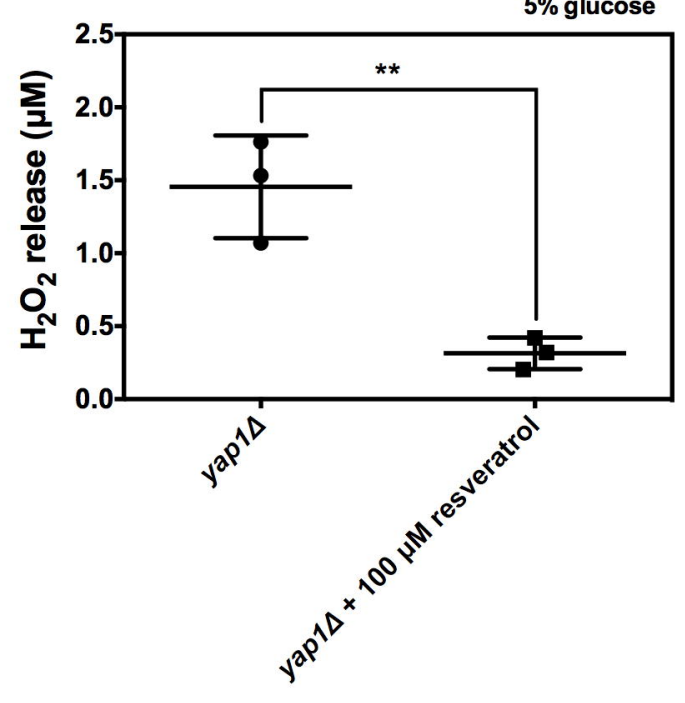

f)

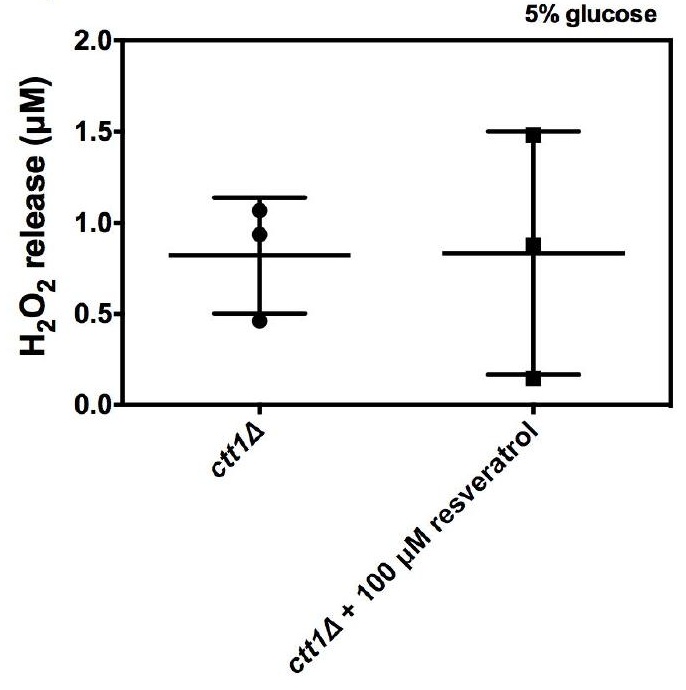


$5 \%$ glucose

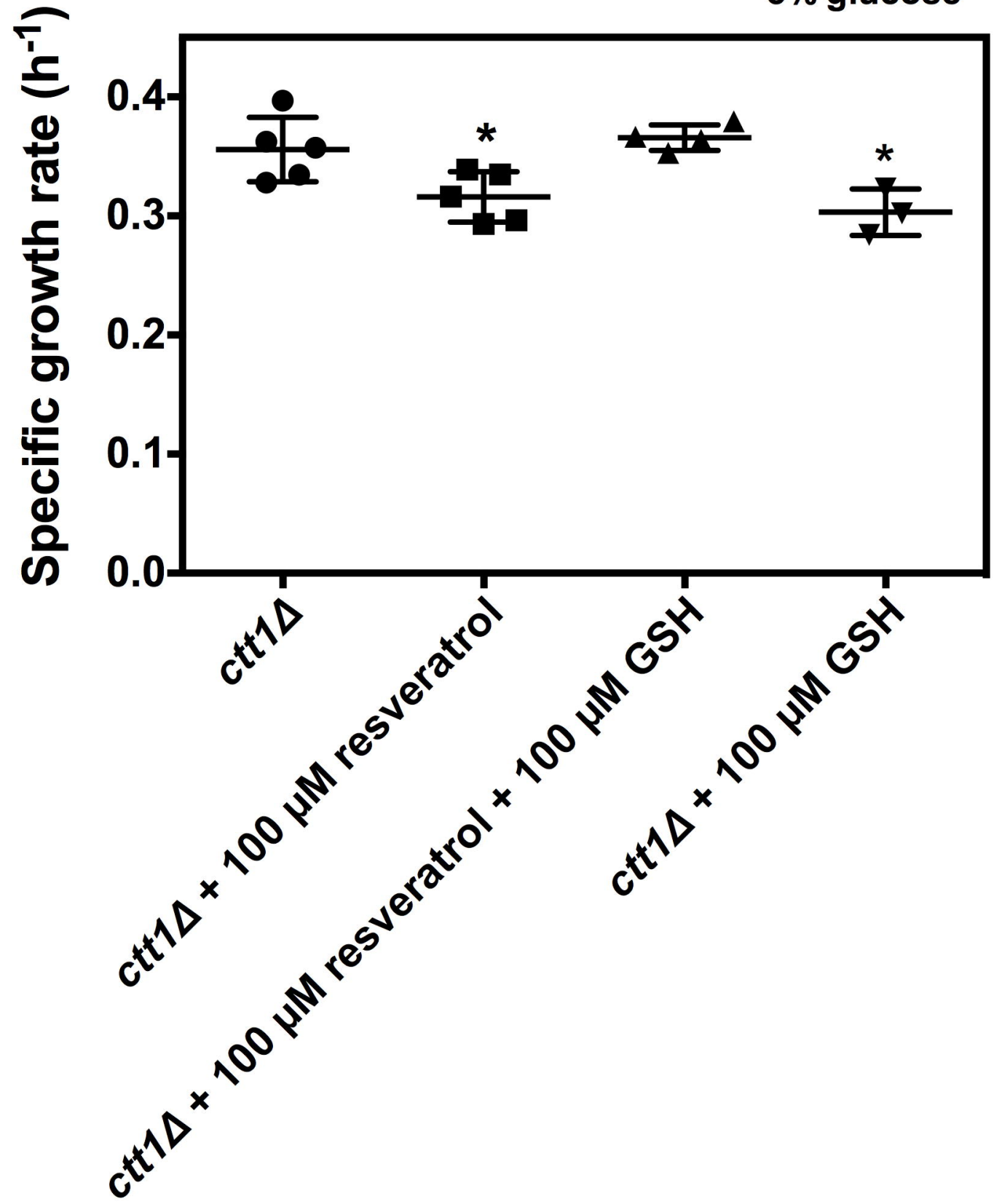


a)

$\mathbf{5 \%}$ glucose

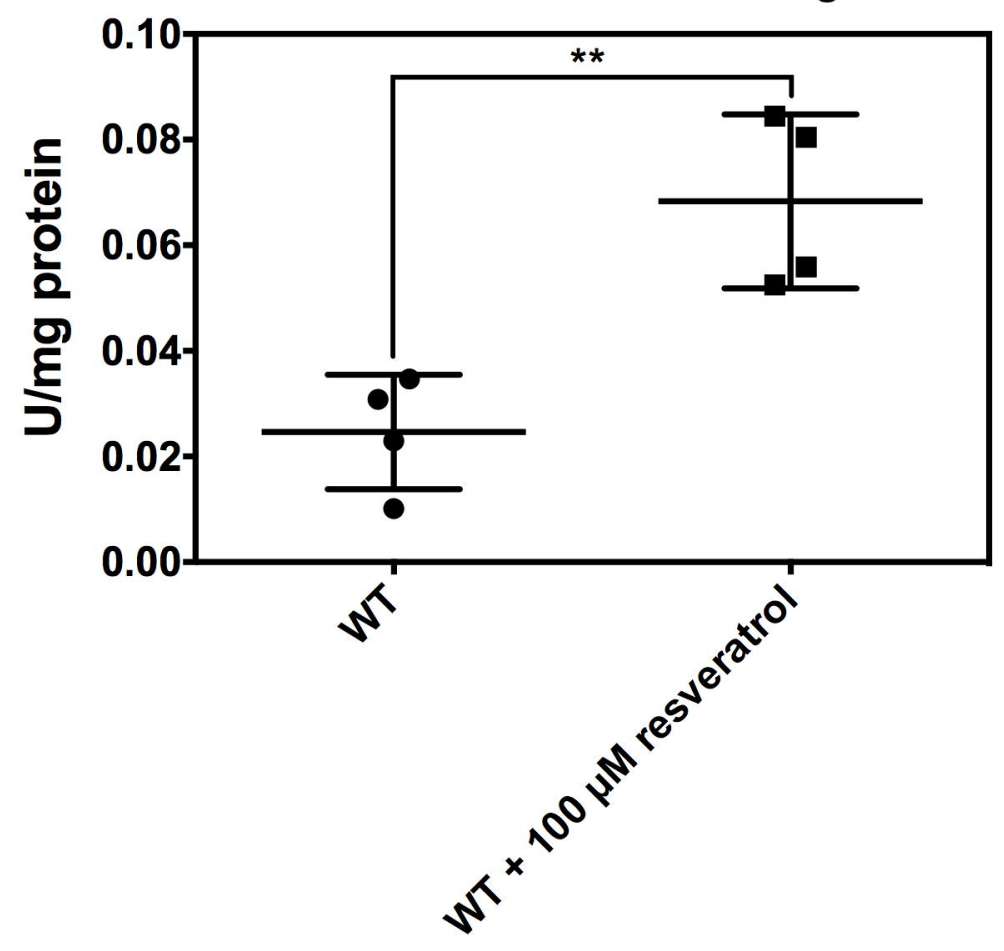

b)

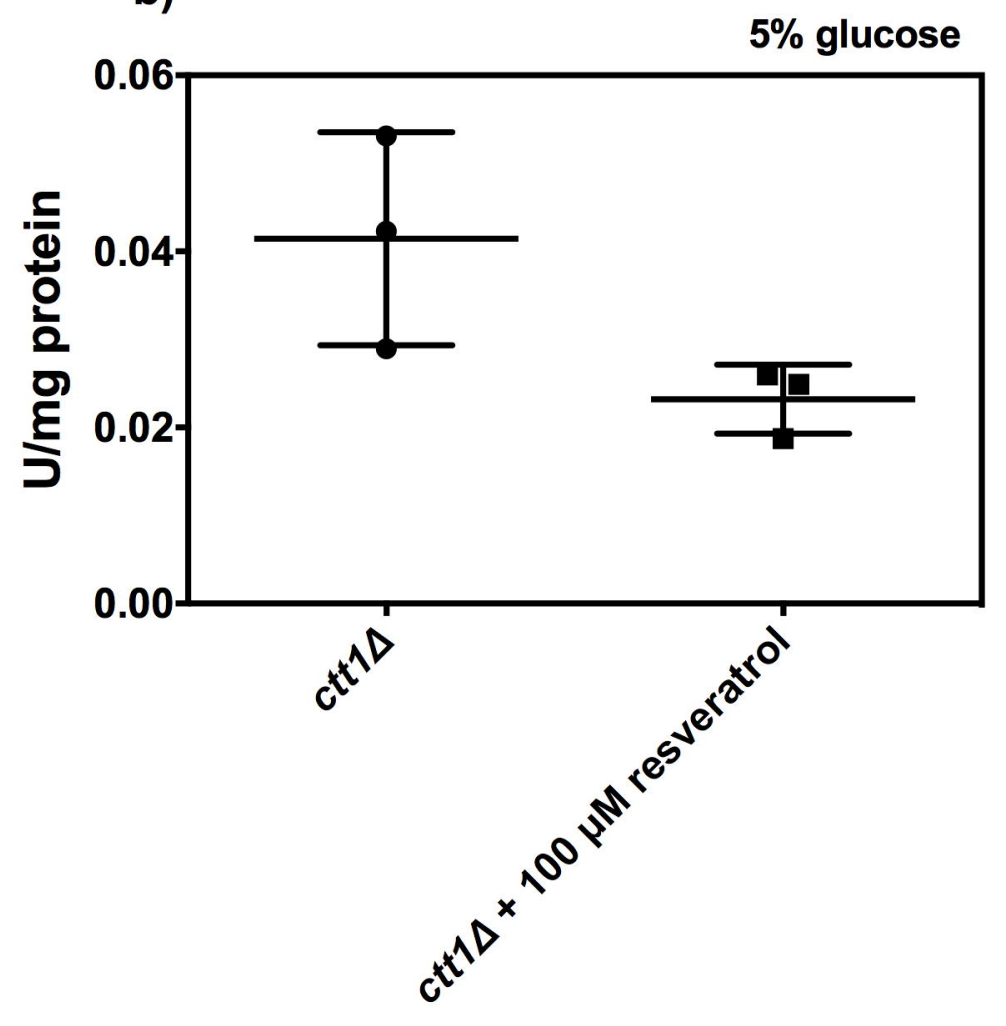

